\title{
FEM Numerical Simulations to Predict the Vibration Reduction Index of Traditional and Lightweight Building Junctions
}

\author{
Arianna Astolfi ${ }^{1}$, Elena Caccherano ${ }^{2}$, Alessio Carullo ${ }^{3}$, Antonella Castellana ${ }^{3}$, Alessia Griginis ${ }^{4}$, \\ Marco Masoero ${ }^{1}$, Giuseppina Emma Puglisi ${ }^{1}$, Louena Shtrepi ${ }^{1}$ \\ ${ }^{1}$ Department of Energy, Politecnico di Torino, Italy; \\ ${ }^{2}$ Cantene S.r.l., Torino, Italy; \\ ${ }^{3}$ Department of Electronics and Telecommunications, Politecnico di Torino, Italy; \\ ${ }^{4}$ ONLECO S.r.l., Torino, Italy
}

\begin{abstract}
This work concerns the use of FEM (Finite Element Methods) software to predict the vibration reduction index $K_{i j}$ of several T-shaped junctions of both traditional heavy and lightweight structures. Much has been done so far with respect to FEM simulations of thermal bridges, but such methodology is not yet widespread in the field of acoustics. Research is needed to provide simplified formulas to be included in the new releases of the EN ISO 12354-1 standard, to overcome discrepancies between calculated and measured values. This should be done particularly for heavy junctions and combinations of heavy and lightweight junctions. In this study a validation of the simulation method has been shown, after its application to simple $\mathrm{T}$-joints typical of traditional building constructions. The intention in the future is to use numerical simulations to provide data or formulas on $K_{i j}$ for more complex types of junctions and to validate simulations with in-laboratory measurements.
\end{abstract}

\section{Introduction}

\section{Measurement and prediction of $K_{i j}$}

In the field of construction, in addition to structural and thermal design, sound insulation design has gained a crucial role as strict standards have been introduced in Europe in the field of outdoor noise protection (Directive 2002/49/EU, 2002).

Between two adjacent rooms, sound travels via the separating element, i.e. by direct transmission, but also through the many other building elements that are connected to the separating wall. This indirect transmission is indicated as flanking transmission and it is often critical in determining the in-situ sound insulation (Hopkins, 2007). In the European Standards EN ISO 12354 (2017), for the estimation of acoustic performance of buildings, and EN 10848 (2017), for the laboratory and in-situ measurement of flanking transmission in buildings, the vibration reduction index, $K_{i j}$, was introduced as a descriptor of the transmission of vibration waves across a junction of walls. It is defined according to the following expression:

$$
K_{i j}=\overline{D_{v, l j}}+10 \log \left(\frac{l_{i j}}{\sqrt{a_{i} a_{j}}}\right)
$$

Where $l_{i j}$ is the common length of the junction between element $i$ and $j$, in meters, $\overline{D_{v, l}}$ is the direction-averaged vibration level difference, in decibels, and $a$ is the equivalent absorption length of the junction $i$ and $j$, in meters. The direction-averaged vibration level difference, $\overline{D_{v, l]}}$, is obtained as:

$$
\overline{D_{v, l j}}=\frac{D_{v, i j}+D_{v, j i}}{2}
$$

Where $D_{v, i j}$ is the velocity level difference between the source element $i$ and the receiver element $j$, in decibels and vice versa for $D_{v, j i}$.

The equivalent absorption length of the element $i, a_{i}$, in meters, is calculated from:

$$
a=\frac{2.2 \pi^{2} S}{c_{0} T_{s}} \sqrt{\frac{f_{\text {ref }}}{f}}
$$

Where $S$ is the area of element $i$ or $j$, in square meters, $T_{s}$ is the structural reverberation time of the element $i$ or $j$, in seconds, $f$ is the octave or one-third octave band center frequency, in Hertz, $c_{0}$ is the speed of sound in air, in meters per second, and $f_{r e f}$ is the reference frequency of 1 $\mathrm{kHz} . T_{s}$ can be calculated as:

$$
T_{s}=\frac{2.2}{f \eta_{\text {tot }}}
$$

Where $\eta_{\text {tot }}$ is the total loss factor, which includes the internal loss factor, the losses due to radiation and the losses at the perimeter of the element. The in-situ total loss factor can be estimated as:

$$
\eta_{t o t} \approx 0.01+\frac{c}{\sqrt{f}}
$$

Where $c$ is a constant depending on the building system. For masonry or concrete elements connected to other heavy elements, $c$ can be set equal to 1 .

The equivalent absorption length is the length of a fictional totally-absorbing edge of an element if its critical frequency is assumed to be $1 \mathrm{kHz}$, giving the same loss as the total losses of the element in a given situation.

Due to the corrections for absorption and dimensions of the structures, $K_{i j}$ becomes invariant, i.e. only depending on the type of junction and the materials and thickness of the structures.

A reliable estimation of the vibration reduction index $K_{i j}$ is crucial for the $i n$-situ evaluation of the apparent sound 
reduction index, $R^{\prime}$, and for the normalized impact sound pressure level, $L^{\prime}{ }_{n}$, when the respective laboratory data $R$ and $L_{n}$ are available (Gerretsen, 1986).

Annex E.3 of EN ISO 12354-1 (2017) provides empirical formulas for the prediction of the frequency independent $K_{i j}$ values in the case of rigid junctions, typical of heavy homogeneous plates, and frequency dependent values between lightweight double leaf walls and homogeneous elements. These values are based on the mass-ratio at the joint, i.e. on the quantity $M$, defined as:

$$
M=\log \left(\frac{m^{\prime} \perp i}{m^{\prime} i}\right)
$$

Where $m_{i}^{\prime}$ is the mass per unit area $\left(\mathrm{kg} / \mathrm{m}^{2}\right)$ of the element $i$ in the transmission path $i j$, and $m_{\perp i}^{\prime}$ is the mass per unit area $\left(\mathrm{kg} / \mathrm{m}^{2}\right)$ of the perpendicular element to $i$ at the junction.

Annex $\mathrm{F}$ provides formulas for junctions between lightweight elements, both characterized in terms of $K_{i j}$ or directly in terms of $\overline{D_{v, l]}}$.

Different shapes of junction are considered in the standard, that are L-, T- and X-shapes.

In the EN ISO 12354 -1 (2017), in addition to Annex E.3, which provides frequency independent $K_{i j}$ formulas for rigid joints, typical of heavy constructions, based on mass-ratio at the joint, the new Annex E. 4 has been added that provides $K_{i j}$ formulas for heavyweight walls in three different frequency ranges (low-frequency range, $50 \mathrm{~Hz}$ to $200 \mathrm{~Hz}$, mid-frequency range, $250 \mathrm{~Hz}$ to $1 \mathrm{kHz}$, highfrequency range, $1.25 \mathrm{kHz}$ to $5 \mathrm{kHz}$ ), derived from numerical simulations.

The new formulas are based on the $P C$ ratio, which is the ratio of the characteristic moment-impedance, as a more relevant quantity than $M$ in the prediction of $K_{i j}$ data. $P C$ ratio equals the ratio $\Psi / \chi$ in Crispin et al. (2014). It is defined as follows:

$$
P C \text { ratio }=\frac{m^{\prime} \perp i}{m^{\prime} i}\left(\frac{f_{c, i}}{f_{c, \perp i}}\right)^{3 / 2}
$$

Where $f_{c, i}, f_{c, \perp i}$ is the critical frequency in $\mathrm{Hz}$, of the element $i$ and of the element perpendicular to $i$ in the $i j$ transmission path.

The critical frequency is calculated as:

$$
f_{c}=\frac{c_{0}^{2}}{\pi} \sqrt{\frac{3 m^{\prime}\left(1-v^{2}\right)}{E h^{3}}}=\frac{c_{0}^{2} \sqrt{3}}{\pi h c_{L}}
$$

Where $h$ is the plate thickness, in meters, $E$ is the Young modulus, in Newton per square meters, $v$ is the Poisson ratio, and $c_{L}$ is the quasi-longitudinal wave speed, in meters per seconds.

The structure-borne power transmission factor, $\gamma i j$, is then calculated as a function of the $P C$ ratio from formulas in Table E.1 of the EN ISO 12354-1 (2017), and then $K_{i j}$ as follows:

$K_{i j}=-10 \lg \gamma_{i j}+5 \lg \frac{f_{c, j}}{f_{\text {ref }}}=-10 \lg \gamma_{j i}+5 \lg \frac{f_{c, i}}{f_{\text {ref }}}$

Where $f_{\text {ref }}$ is the reference frequency of $1 \mathrm{kHz}$.
Such an update of the Annex E has been considered necessary since many researchers found discrepancies between their findings and the empirical simplified predictions (Schiavi and Astolfi, 2005; Crispin et al. 2006).

\section{Background on the prediction formulas and outline of the work}

The approach followed by the frequency-independent empirical formulas in Annex E.3 of EN ISO 12354-1 (2017), for heavy rigid junctions, considers only incident and transmitted bending waves through the junction, while a possible generation of in-plane waves is not considered. The generation of in-plane waves at the junction means that the resulting transmission coefficient tends to vary with frequency, and in particular to affect mid and high frequency ranges. To overcome such a limitation, Hopkins (2014) used wave theory to determine the vibration reduction index $K_{i j}$ considering both "bending only" and "bending and in-plane" waves transmission for several L, T-, X-junctions made of different heavyweight materials with different thicknesses. Regression analysis for the wave theory $K_{i j}$ data was carried out for each junction using the quantity $M$ described in (6). Results showed the dependence of $K_{i j}$ on frequency and the need of new empirical curves for junctions of solid heavyweight walls/floors.

These results have been confirmed by Publet-Puig and Guigou-Carter (2015), who performed both Finite Element Method (FEM) and Spectral FEM (SFEM) simulations to provide data and guidelines oriented to the EN-12354 design method for flanking transmission in the case of heavy-weight buildings.

Crispin et al. (2014) introduced the ratio of the characteristic moment-impedance $\Psi / \chi$ as a more relevant quantity than $M$ in the prediction formulas of $K_{i j}$ data. They used FEM simulations, which were validated with measured data, and derived new prediction formulas for $\mathrm{T}$-rigid junctions and $\mathrm{X}$-rigid junctions.

Other recent studies based on numerical simulations have deepened the new quantity $\Psi / \chi$ as parameter to properly predict $K_{i j}$ data in a great number of heavy-junctions (Hopkins et al., 2016; Publet-Puig and Guigou-Carter, 2017). These works constitute the major contribution to the new paragraph (E.4) included in Annex E in EN 12354 (2017). However, not all the estimations obtained by means of numerical simulations have been verified with measurements in real junctions. In fact, in the existing literature a limited number of studies dealt with laboratory measurements of $K_{i j}$. Crispin et al. (2004 and 2006) presented the data measured on both rigid junctions and junctions with flexible interlayer made of cellular concrete and/or concrete blocks using flanking laboratories. They also compared $K_{i j}$ measures with the prediction methods of the old release of the standard EN 12354-1 (2000) and of the SEA model and they found preliminary discrepancies that have brought to the new parameter $\Psi / \chi$ proposed in the above-mentioned Crispin et al. (2014). 
In order to further provide formulas for $K_{i j}$ calculation in the case of different building typologies and different junction shapes, to be used by practitioners, a further step is needed, which comprehends the simulation of $K_{i j}$ for many possible junctions of heavyweight and lightweight walls. The latter have not been deepened in literature so well. These simulations should be verified with laboratory measurements according to the EN 10848 standard (2017) at least for some typical configurations, including the new insights from Hopkins and Robinson (2014).

To fill in such a gap in the existing literature, the aim of this work is to use FEM simulation to match the new frequency dependent $K_{i j}$ values obtained with the new formulas in the Annex E.4 of the standard, for simple Tshaped junctions, which belong to the traditional way of construction. Particularly, heavyweight walls made of concrete and masonry and a combination of heavyweight walls and a lightweight element, i.e. a double leaf plasterboard wall, have been considered.

For each junction, $K_{i j}$ values were evaluated through FEM simulations and compared with the predictions according to both Annex E.3 and E.4 of EN ISO 12354-1 (2017). Half- scaled T-junctions were considered. The half-scaled model complied with the theoretical background as discussed by Kling (2007) and Crispin et al. (2017) and reduced the simulation times.

\section{FEM analysis}

\section{Description of the model}

FEM analysis was carried out in order to determine the velocity level difference $D_{v, i j}$ and $D_{v, j i}$ from which $K_{i j}$ has been calculated according to formula (1). The analysis was performed using the software COMSOL $\AA$, in particular the shell interfaces of the structural mechanics module. Different T-junctions were modelled varying the materials they were made up. The study is set in the frequency domain.

A shell is a thin-walled structure in $3 \mathrm{D}$ where a simple form is assumed for the variation of the displacement through the thickness. In a shell model it is necessary to provide the thickness, a possible offset and the elastic material properties. In this study the shell models were made considering the faces at the midplane of the real geometry (see Figure 1). No offset was provided.

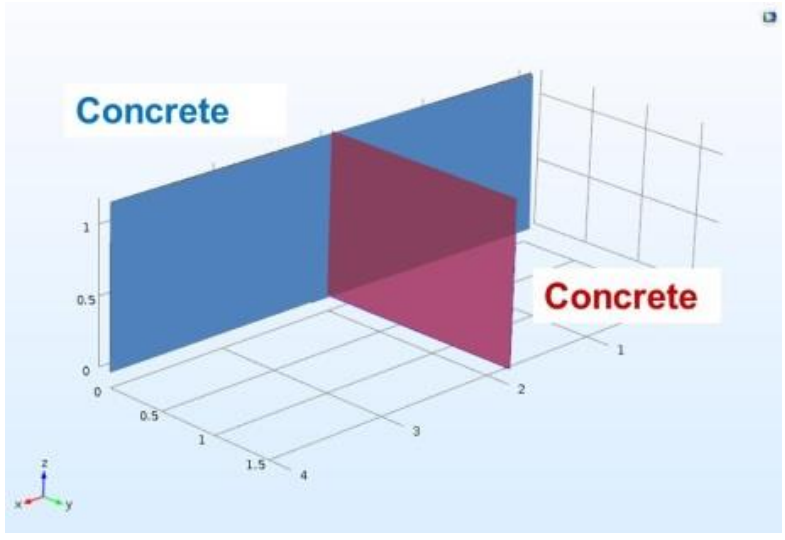

Figure 1: Example of a 3D shell model of a T-junction.
The plates of the junction are excited one at a time through the rain on the roof excitation (Hopkins, 2007). A face load is provided to the surface of the plate to be excited (Figure 2). The load is defined as force per unit area. The force has unity magnitude and random phase.

As shown in Figure 3, all the plate boundaries are set as free boundaries except for the sides connected with the floor, which are simply supported (Hopkins, 2007). The floor is not included in the model. The joint line is a free (unconstrained) node. According to Hopkins (2003), this setting allows generation of both bending and in-plane waves at medium and higher frequencies.

The FEM model is based on the assumption of thin plate theory. At frequencies above the thin plate limit for concrete/masonry walls, which are around $1 \mathrm{kHz}$ (Hopkins, 2007), thick plates behavior with shear deformation and rotatory inertia may occur. However, the comparison of measured and FEM predicted data does not indicate that large errors were introduced with the thinwall approximation (Hopkins, 2003).

The temporal and spatial average mean-square velocity over the surfaces has been obtained over a free triangular mesh. Mesh elements have dimensions lower than $\lambda / 6$ (where $\lambda$ is the smallest wavelength of interest for the study), according to Hopkins (2003) and Crispin et al. (2014). The mesh of the shell model counts $\approx 4000$ elements.

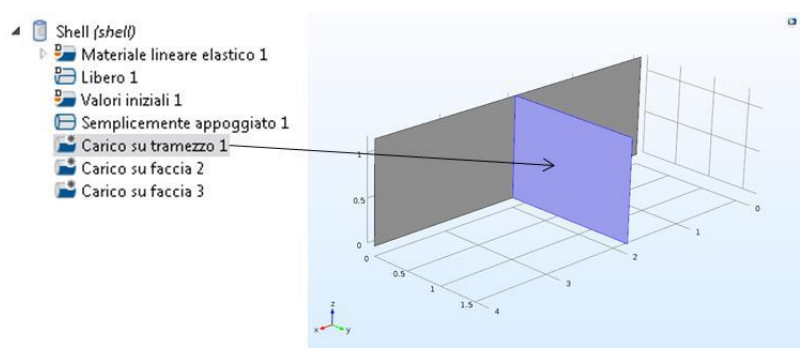

Figure 2: Face load on a plate of the T-junction in the shell model.

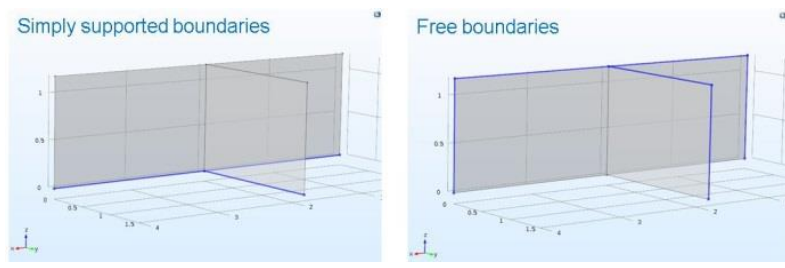

Figure 3: Boundary conditions for the T-junction shell model.

The frequency range covered one-third octave bands between $250 \mathrm{~Hz}$ and $6300 \mathrm{~Hz}$. Three $K_{i j}$ values, corresponding to three frequencies in each band, were calculated and averaged. This average value was assigned to the central frequency of the band to give a single value representing the band.

The frequency range $250-6300 \mathrm{~Hz}$ relates to the half-scale model. In order to refer to the full-scale model frequency 
range, frequencies should be halved, i.e. results at $500 \mathrm{~Hz}$ for half-scale correspond to $250 \mathrm{~Hz}$ in full-scale.

In a miniaturized facility, scaling all the dimensions of the wall by a factor 2 ensures that the structure-borne sound fields are similar when the frequency is increased by 2 (Crispin et al., 2017). This can be confirmed if the ratio between the Young's modulus and the material density as well as the internal damping do not change along frequencies (Kling, 2007). The main assumptions that have been made in this work is that the Young's modulus and the internal loss factor of the half-scaled model in the model frequency range match the Young's modulus and internal loss factor of the full-size facility, in the full-size frequency range.

Table 1 and Figure 4 show the characteristics and the dimensions of the half-scaled nodes, respectively. Tjunctions have been considered between conventional, lightweight and reinforced concrete walls, double leaf plasterboard and brick holed walls.

The wall thickness in real scale was determined to respect the minimum requirement for dwellings of the Apparent Sound Reduction Index, $R$ ', , between rooms, equal to 50 $\mathrm{dB}$, according to the 24 European Countries (Rasmussen, 2010). Calculations were made of $R_{w}$, according to the single-index method of the EN ISO 12354-1 (2017), assuming as external wall and internal walls the layer identified as $i$ and $j$ in Figure 4, respectively.

Table 1: Characteristics of the T-junctions. The following abbreviations are used: CONV. for Conventional concrete, PL. for Plasterboard, RE. for Reinforced concrete. LI. for Lightweight concrete, BRICKS for Brick holed.

\begin{tabular}{|c|c|c|c|}
\hline ID & Layer & Material & Depth $(\mathrm{m})$ \\
\hline \multirow{2}{*}{1} & external & CONV. & 0.1 \\
\cline { 2 - 4 } & internal & CONV. & 0.1 \\
\hline \multirow{2}{*}{2} & external & CONV. & 0.1 \\
\cline { 2 - 4 } & internal & PL. & 0.09 \\
\hline \multirow{2}{*}{3} & external & RE. & 0.1 \\
\cline { 2 - 4 } & internal & LI. & 0.1 \\
\hline \multirow{2}{*}{4} & external & BRICKS & 0.15 \\
\cline { 2 - 4 } & internal & BRICKS & 0.15 \\
\hline
\end{tabular}

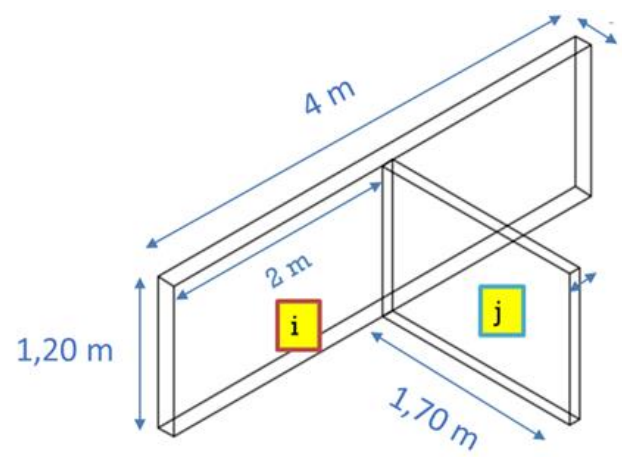

Figure 4: Dimension of the half-scaled model of one of the simulated T-junction.
The damping of the structure is taken into account through the isotropic loss factor of the material. Table 2 shows all the materials properties. Data are mostly derived from literature and, in the case of the concrete layers, from measurements performed in previous experiments. The internal loss factor instead of the in-situ total loss factor in (5) was applied in the model as first approximation (EN ISO 12354-1, 2017). An EPS coat could have been added to the $\mathrm{T}$-junctions in order to simulate a real façade covered with a thermal insulation layer, but its influence on $K_{i j}$ has been considered negligible.

Table 2: Materials properties of the walls.

\begin{tabular}{|c|c|c|c|c|}
\hline Material & $\begin{array}{c}\text { Density } \\
\left(\mathrm{kg} / \mathrm{m}^{3}\right)\end{array}$ & $\begin{array}{c}\text { Young } \\
\text { modulus } \\
(\mathrm{GPa})\end{array}$ & $\begin{array}{c}\text { Poisson } \\
\text { ratio }\end{array}$ & $\begin{array}{c}\text { Internal } \\
\text { loss } \\
\text { factor }\end{array}$ \\
\hline $\begin{array}{c}\text { Conventional } \\
\text { concrete } \\
\text { (CONV.) }\end{array}$ & 2400 & 30 & 0.2 & 0.01 \\
\hline $\begin{array}{c}\text { Lightweight } \\
\text { concrete (LI.) }\end{array}$ & 1450 & 15 & 0.2 & 0.01 \\
\hline $\begin{array}{c}\text { Reinforced } \\
\text { concrete } \\
\text { (RE.) }\end{array}$ & 2200 & 41 & 0.2 & 0.01 \\
\hline $\begin{array}{c}\text { Brick holed } \\
\text { (BRICKS) }\end{array}$ & 1500 & 10.5 & 0.2 & 0.01 \\
\hline $\begin{array}{c}\text { Plasterboard } \\
\text { (PL.) }\end{array}$ & 860 & 1.7 & 0.3 & 0.0141 \\
\hline $\begin{array}{c}\text { Steel } \\
\text { (ST.) }\end{array}$ & 7800 & 199 & 0.28 & 0.0001 \\
\hline $\begin{array}{c}\text { Fiberglass } \\
\text { (FB.) }\end{array}$ & 36 & 0.2 & 0.3 & 0.18 \\
\hline
\end{tabular}

The T-junction n. 2 is intended between an external homogeneous wall made of conventional concrete and a plasterboard wall. The plasterboard wall was double leaf. It consisted of two leaves of plasterboard (single layer 195 $\mathrm{mm}$ thick) and a single metal frame with a cavity depth of $50 \mathrm{~mm}$ (single metal frame $6 \mathrm{~mm}$ thick) filled with $50 \mathrm{~mm}$ mineral wool. All the sizes are related to the half-scale model. The metal frame was modelled as a series of 7 vertical metal laminates, normal to the plasterboard leaves at a fixed mutual distance, and 2 horizontal laminates, one at bottom and one on the top of the structure. The layer of fiberglass was modelled by a vertical plane between the plasterboard walls.

As far as the brick wall is concerned, it has been modelled as a thin homogeneous isotropic plate. Walls made of blocks, bricks, and slabs are not homogeneous and isotropic due to the hollows inside them and the mortared joints (Dijckmans and Vermeir, 2013; Santoni et al., 2017; Santoni et al., 2019). Anyway, they can be considered as first approximation homogeneous if the dimensions of the holes are small and the volume of holes is less than $15 \%$ of the gross volume (EN 12354-1, 2017). Moreover, for cast in situ block/brick walls with horizontal and vertical joints mortared, the bending stiffness is not usually more than $20 \%$ higher in one 
direction than the other and thus it is reasonable to treat these walls as isotropic (Hopkins, 2007).

\section{Validation of the model}

A FEM shell model of the $\mathrm{H}$-junction reported in Hopkins (2003) was created and then validated through comparison with $D_{i j}$ measured by Hopkins. Figure 5 shows how the $D_{i j}$ results of our model fit the values obtained in the reference for most of the frequency range. Dissimilarities can be attributed to details in the modelling such as mesh, element type, excitation points, and post-processing, as underlined by Hopkins et al. (2016).

The current study was performed in half-scale. Figure 6 shows the comparison between the $K_{i j}$ values obtained in real-scale and in half-scale for a same simulated $\mathrm{T}$ junction. A good matching has been obtained for the two simulations, which has brought to the use of half-scaled models for the rest of the study.

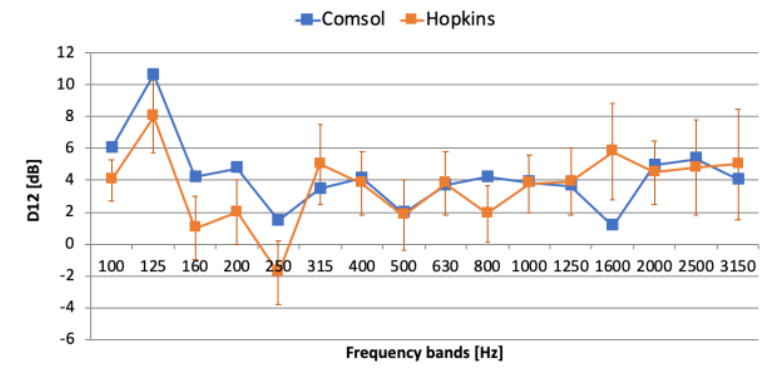

Figure 5: Comparison between $D_{i j}$ obtained by Hopkins (2013) (red line) and by a shell model created with COMSOL®(blu line) for the same junction.

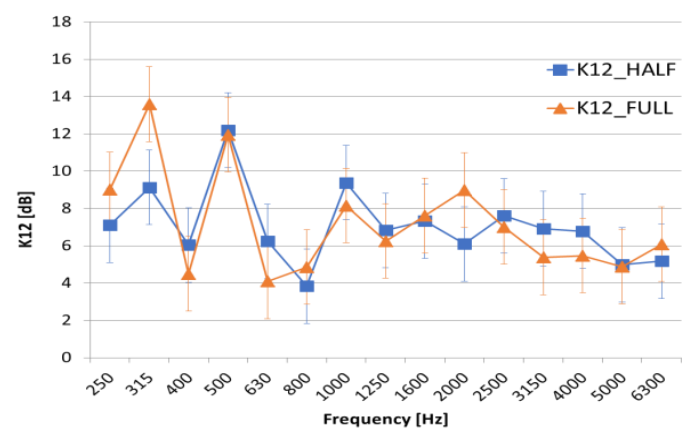

Figure 6: Comparison of $K_{i j}$ values in real and half scale models for the same T-junction realised with a shell

FEM structure. Half-scale results are halved in

frequency from to 200-6300 to $100-3150 \mathrm{~Hz}$ in order to be comparable to full-scale ones.

\section{Results and discussion}

Figures from 7 to 10 show the one-third octave band $K_{i j}$ values for the transmission around the corner (K12) and across the straight section (K23), obtained from FEM simulation of the four T-junctions described in Tables 1 and 2. $K_{i j}$ values are also compared to those calculated according to the formulas in paragraph E.3.3 of the Annexes E.3 (A in the figures) and in Table E.1 of the
Annex E.4 (B in the figures) of the standard EN ISO 12354-1 (2017). The frequency range $250-6300 \mathrm{~Hz}$ relates to the half-scale model.

Kij formulas in the Annex E.3 are based on empirical assumptions and are given with dependency on the mass per unit area of the elements connected at the junction, as shown in formula (6). According to the Annex E.3, Kij formulas for junctions between rigid junctions, typical of heavy homogeneous plates, are frequency independent, while those between homogeneous elements and lightweight double leaf walls are frequency dependent. Frequency dependent $K i j$ values are provided in the Annex E.4 for junctions between homogeneous, isotropic plates, that are based on numerical simulations. The formulas in Annex E.4 have been also validated with inlaboratory measurements (Hopkins et al., 2016).

From the results it can be seen that the frequency behaviour is very similar to that provided from the Annex E.4 of the EN ISO 12354-1 (2017) for all the T-junctions and for both the transmission paths. Lower values are displayed at higher frequencies for the K23 path in the case of heavy constructions, while for the same constructions a more constant frequency trend is highlighted for K12 path. In the case of joint between homogeneous elements and lightweight double leaf walls, K23 path shows higher values at lower frequencies, while a tendency to have increasing $K_{i j}$ values with frequency is shown for the K12 path.

For transmission across the straight section of the heavy joints, there was a decrease in transmission loss with increasing frequency, which is confirmed in real measurements as indicative of in-plane wave generation at the junction, as underlined by Hopkins (2014).
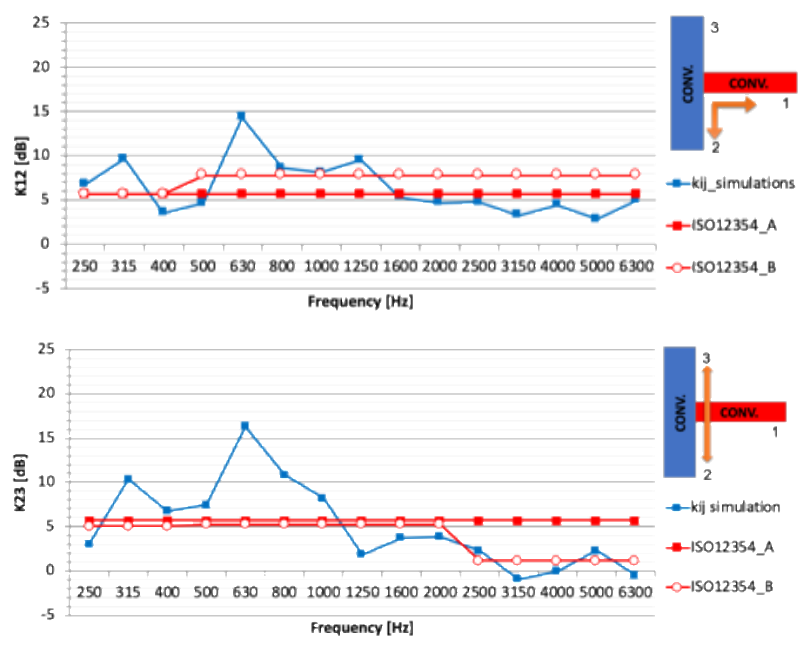

Figure 7: Simulated $K_{i j}$ values for the transmission across the corner (upper chart) and across the straight section (lower chart) of a T-junction made of two $10 \mathrm{~cm}$ conventional concrete layers. 


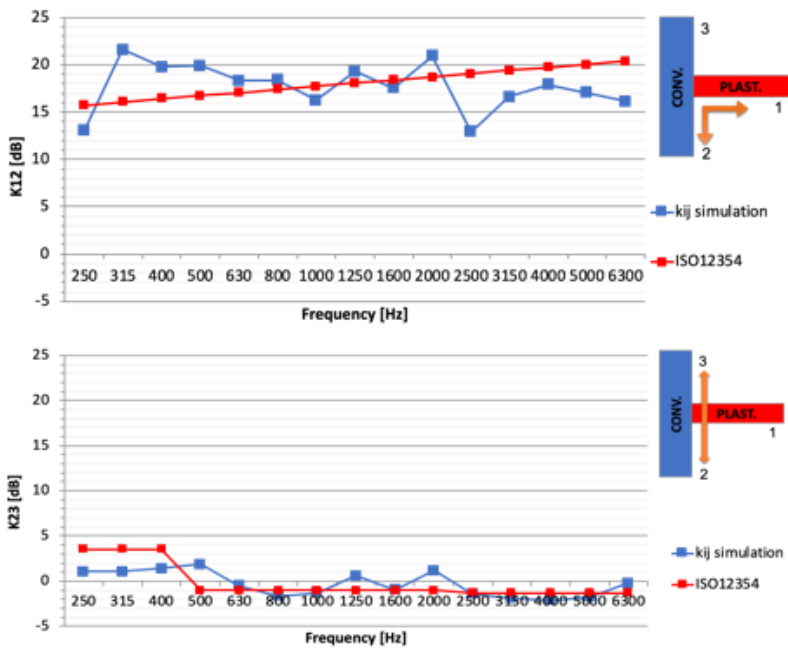

Figure 8: As in Figure 7, but the T-junction is made of $10 \mathrm{~cm}$ conventional concrete layer and $9 \mathrm{~cm}$ double leaf plasterboard wall.
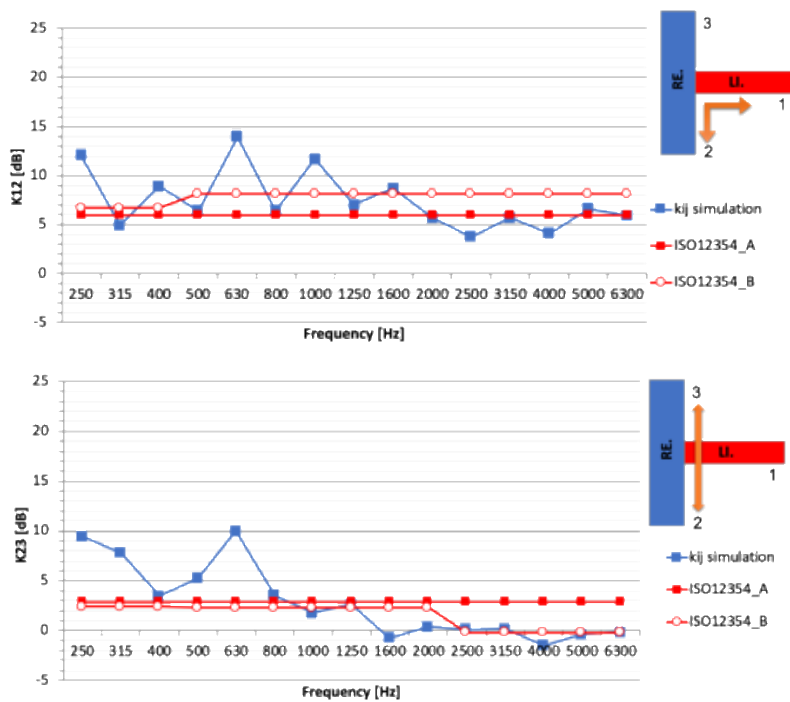

Figure 9: As in Figure 7, but the T-junction is made of $10 \mathrm{~cm}$ reinforced concrete layer and $10 \mathrm{~cm}$ lightweight concrete layer.

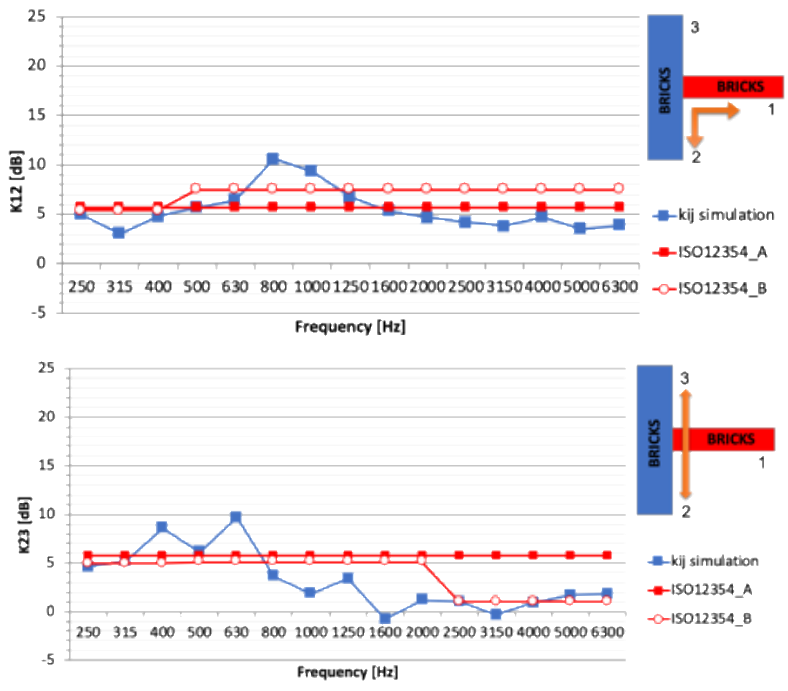

Figure 10: As in Figure 7, but the T-junction is made of two $15 \mathrm{~cm}$ brick layers.
Figure 11 shows the mean values of $\mathrm{K}_{\mathrm{ij}}$, from $250 \mathrm{~Hz}$ to $6.3 \mathrm{kHz}$ one-third octave bands, in the case of transmission paths $\mathrm{K} 12$ and $\mathrm{K} 23$, for the T-junctions from 1 to 4 in Table 1 . The highest value of about $18 \mathrm{~dB}$ is shown in the case of transmission around the corner for the node 2 , where a conventional concrete wall is joined to a double leaf plasterboard wall. On the opposite, a very low value is found in the case of the same joint for the transmission across the straight section. For the other three nodes, compatible $\mathrm{K}_{\mathrm{ij}}$ of about $3 \div 7 \mathrm{~dB}$ have been found for both the transmission paths.

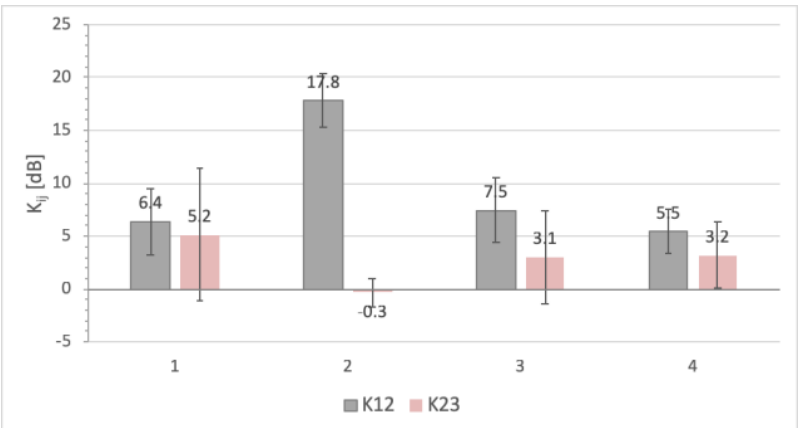

Figure 11: Mean value over the one-third octave band frequencies of the simulated $K_{i j}$, for the transmission across the corner (K12) and across the straight section (K23) of the T-junctions described in Table 1.

Figures 12 and 13 show the $K_{i j}$ mean values of the Tjunctions, for the three ranges of low, medium and high frequencies, in the case of transmission paths K12 and $\mathrm{K} 23$. As far as K12 path is concerned, compatibility among the three frequency ranges is shown for all the nodes with, as underlined above, higher values for node 2 of about $16 \div 18 \mathrm{~dB}$ and lower values of about $5 \div 7 \mathrm{~dB}$ for the other nodes. K23 path shows higher vibration transmission at the higher frequencies, and generally lower $K_{i j}$ values for all the frequency ranges compared to K12. The lowest $K_{i j}$ value is for node 2.

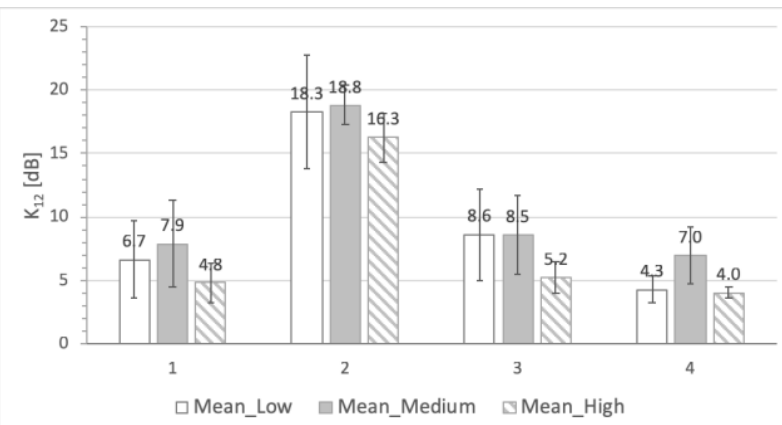

Figure 12: Mean values over the low $(250 \mathrm{~Hz}-400 \mathrm{~Hz})$, medium $(500 \mathrm{~Hz}-2 \mathrm{kHz})$ and high $(2.5 \mathrm{kHz}-6.3 \mathrm{kHz})$ frequencies, of the simulated $K_{i j}$ for the transmission across the corner of the T-junctions in Tables 1 and 2. 


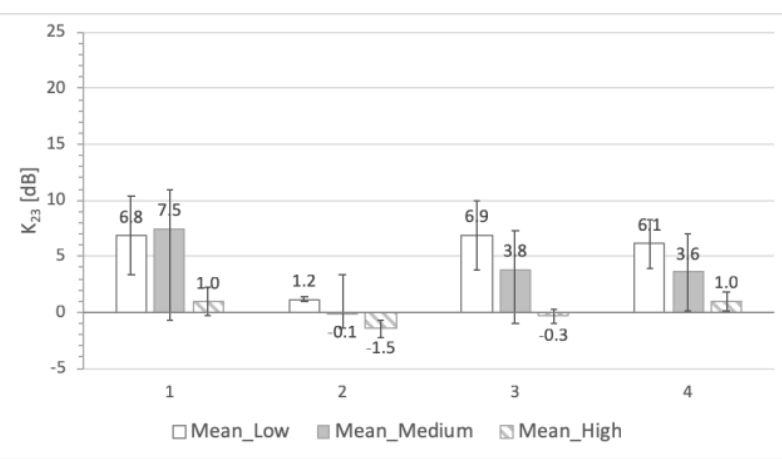

Figure 13: As in Figure 12, but for the simulated $K_{i j}$ the transmission is across the straight section of the $T$ junctions in Tables 1 and 2.

A good matching between simulated $K_{i j}$ values and calculated values according to the frequency dependent formulas in EN ISO 12354 -1, has been found, as shown in Figures 14 and 15, for K12 and K23, respectively, in the three frequency ranges. In particular, results are compatible among them for both the transmission paths and the frequency ranges, suggesting the application of FEM simulation to investigate more complex junction geometries.

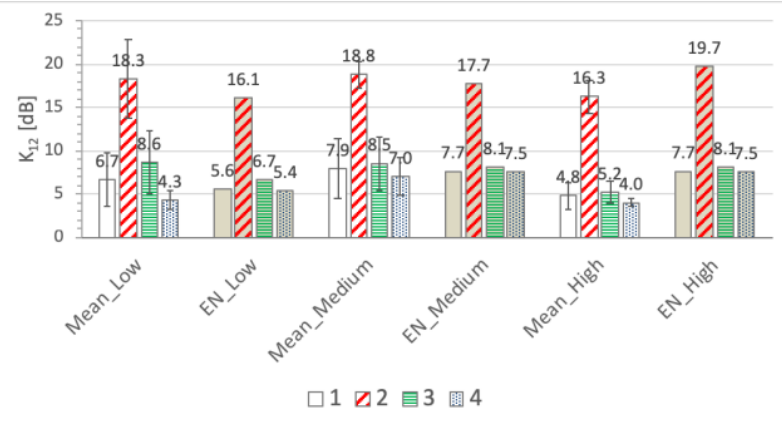

Figure 14: Mean values over the low $(250 \mathrm{~Hz}-400 \mathrm{~Hz})$, medium $(500 \mathrm{~Hz}-2 \mathrm{kHz})$ and high $(2.5 \mathrm{kHz}-6.3 \mathrm{kHz})$

frequencies, of the simulated $K_{i j}$ for the transmission across the corner of the T-junctions from 1 to 4 , described in Tables 1 and 2. The graph also shows the mean values, over the same frequency ranges, of the $K_{i j}$ calculated according to the EN ISO 12354 (1) formulas.

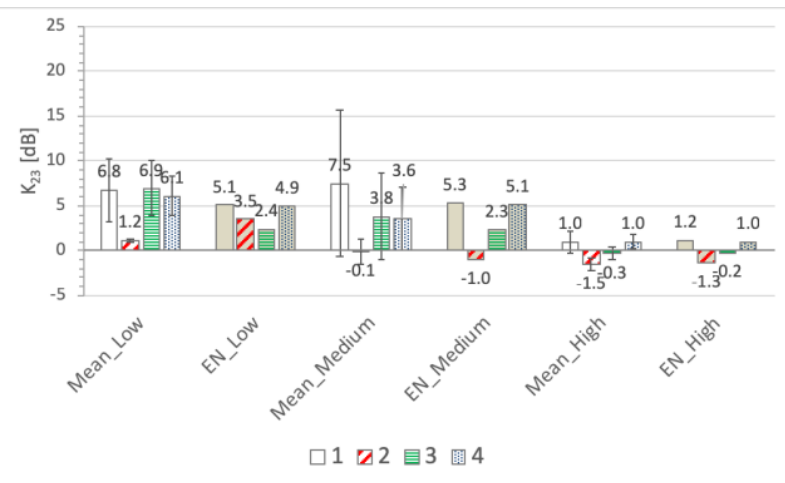

Figure 15: As in Figure 14, but for the simulated $K_{i j}$ the transmission is across the straight section of the $T$ junctions from 1 to 4, described in Tables 1 and 2 .
In general, these results are in good agreement with the measured $K_{i j}$ values obtained by Crispin et al. (2004 and 2006) for similar heavy T-junctions. They found an average $K_{i j}$ value on our medium-frequency range of about $7 \mathrm{~dB}$ for both the K12 and K23 paths, when the two walls had the same depth. Instead, they found higher values close to $16 \mathrm{~dB}$ for $\mathrm{K} 12$ and close to about $1 \mathrm{~dB}$ for $\mathrm{K} 23$, when the two walls had very different masses, with external wall more massive than internal one.

The same good agreement was found with FEM simulations of the same heavy nodes by Poblet-Puig and Guigou-Carter (2015).

Hopkins (2014), in the case of rigid heavy T-junctions, indicates the importance of frequency dependence for $K_{i j}$. In fact, application of formulas in Annex E.3 of the standard EN ISO 12354-1 (2017) that considers the frequency independency of $K_{i j}$ would have brought to an overestimation of the values for transmission across the straight section at higher frequencies (see red lines named ISO12354_A in Figures 8, 10 and 11 lower charts).

\section{Conclusion}

This paper has shown that FEM simulations can be applied to investigate vibration transmission across building junctions in the case of traditional and lightweight constructions. Vibration transmission index, $K_{i j}$, resulted compatible with the validated EN ISO $12354-$ 1 (2017) frequency dependent formulas, for both the transmission around a corner and the straight section, and for all the frequency range. Comparisons will be carried out in the future with in-laboratory measured data according to the EN ISO 10848 standard (2017), of the same nodes and of other more complicated T-junctions.

The FEM models require a frequency-dependent loss factor for the walls, i.e. the total loss factor, which is the loss factor that is likely to occur when heavyweight walls are connected to many other walls and floors in-situ (Hopkins, 2016; EN ISO 12354-1, 2017). Next steps will include this frequency-dependent loss factor in the simulations.

Future work will concern the validation of FEM simulations with in-laboratory measurements, and the implementation of new FEM simulations for the prediction of simple $K_{i j}$ formulas in the case of more complex joints.

\section{Acknowledgement}

The Authors acknowledge Mr G. Volpatti and Mr D. Zampini by CEMEX Research Group AG for the physical data of the concrete used for the simulations.

\section{References}

Crispin, C., Mertens, C., Blasco, M., Ingelaere, B., Van Damme, M., \& Wuyts, D. (2004). The vibration reduction index $\mathrm{K}_{\mathrm{ij}}$ : laboratory measurements versus predictions EN 12354-1 (2000). Proocedings from the 33 rd International Congress and Exposition on Noise Control Engineering, INTER-NOISE 2004. Prague (Czech Republic), 22-25 August 2004. 
Crispin, C., Ingelaere, B., Van Damme, M., \& D. Wuyts (2006). The vibration reduction index $\mathrm{K}_{\mathrm{ij}}$ : laboratory measurements for rigid junctions and for junctions with flexible interlayers. Building Acoustics 13(2), 99111.

Crispin, C., De Geetere, L., \& Ingelaere, B. (2014). Extensions of EN 12354 vibration reduction index expressions by means of FEM calculations. Proocedings from INTER-NOISE 2014. Melbourne (Australia), 16-19 November 2014.

Crispin, C., Mertens, C. \& Dijckmans, A. (2017). Detailed analysis of measurement results of flanking transmission across a junction composed of double walls carried out on a half scaled test bench. Proocedings from the 24th International Congress on Sound and Vibration. London (UK), 23-27 July 2017.

Dijckmans, A. \& Vermeir, G. (2013). Development of a hybrid wave based-transfer matrix model for sound transmission analysis. The Journal of the Acoustical Society of America, 133(4), 2157-2168.

Directive 2002/49/EU of The European Parliament and of The Council of 25 June 2002 relating to the Assessment and Management of Environmental Noise.

EN 12354 (2000). Building acoustics. Estimation of acoustic performance in buildings from the performance of elements. Part 1.

EN ISO 12354 (2017). Building acoustics. Estimation of acoustic performance in buildings from the performance of elements. Part 1.

EN ISO 10848 (2017). Acoustics. Laboratory and field measurement of flanking transmission for airborne, impact and building service equipment sound between adjoining rooms. Parts 1-2-3-4.

Gerretsen, E. (1986). Calculation of airborne and impact sound insulation between dwellings. Applied Acoustics 19(4), 245-264.

Hopkins C. (2003). Vibration transmission between coupled plates using finite element methods and statistical energy analysis. Part 1: Comparison of measured and predicted data for masonry walls with and without apertures. Applied Acoustics 64, 955-973.
Hopkins, C. (2007). Sound Insulation. Elsevier. Amsterdam (NL).

Hopkins, C. (2014). Determination of vibration reduction indices using wave theory for junctions in heavyweight buildings. Acta Acustica united with Acustica 100(6), 1056-1066.

Hopkins, C. \& M. Robinson (2014). On the evaluation of decay curves to determine structural reverberation times for building elements. Acta Acustica united with Acustica 99, 226 - 244.

Hopkins, C., Crispin, C., Poblet-Puig, J., and C. GuigouCarter (2016). Regression curves for vibration transmission across junctions of heavyweight walls and floors based on finite element methods and wave theory. Applied Acoustics 113, 7-21.

Kling, C. (2007). Miniaturising a wall test facility. Building Acoustics 14(4), 243-266.

Poblet-Puig, J. \& C. Guigou-Carter (2015). Using spectral finite elements for parametric analysis of the vibration reduction index of heavy junctions oriented to flanking transmissions and EN-12354 prediction method. Applied Acoustics 99, 8-23.

Poblet-Puig, J. \& C. Guigou-Carter (2017). Catalogue of vibration reduction index formulas for heavy junctions based on numerical simulations. Acta Acustica united with Acustica 103 (4), 624-638.

Rasmussen, B. (2010). Sound insulation between dwellings. Requirements in building regulations in Europe. Applied Acoustics 71(4), 373-385.

Santoni, A., Bonfiglio, P., Davy, J. L., Fausti, P., Pompoli, F., \& Pagnoncelli, L. (2017). Sound transmission loss of ETICS cladding systems considering the structureborne transmission via the mechanical fixings: Numerical prediction model and experimental evaluation. Applied acoustics, 122, 88-97.

Santoni, A., Fausti, P., \& Bonfiglio, P. (2019). Building materials: Influence of physical, mechanical and acoustic properties in sound prediction models. Building Acoustics, 26(1), 3-20.

Schiavi, A. \& A. Astolfi (2010). The prediction of the vibration reduction index Kij for brick and concrete rigid junctions. Applied Acoustics 71(6), 523-530. 